\title{
Simultaneous control of nanocrystal size and nanocrystal-nanocrystal separation in CdS nanocrystal assembly
}

\author{
SAMEER SAPRA and D D SARMA* \\ Solid State and Structural Chemistry Unit, Indian Institute of Science, \\ Bangalore 560 012, India \\ *Also at Jawaharlal Nehru Centre for Advanced Scientific Research, Jakkur P.O., \\ Bangalore 560 064, India \\ E-mail: sarma@sscu.iisc.ernet.in
}

\begin{abstract}
We report an easy, one pot synthesis to prepare ordered CdS nanocrystals with varying inter-particle separation and characterize the particle separation using x-ray diffraction at low and wide angles.
\end{abstract}

Keywords. CdS nanocrystals; self assembly; inter-particle separation.

PACS Nos 81.07.Bc; 81.16.Dn; 81.16.Be

\section{Introduction}

Nowadays methods to synthesize size selective, nearly mono-disperse nanocrystals are available [1-4] for use in many applications such as labels for biological molecules [5], as phosphors [6], in polymer-LED applications [7,8], in polymer electronics $[9]$, and in photovoltaic devices $[10,11]$. Besides these applications which use properties of individual nanocrystals, a host of other applications requires a well-defined connectivity between the nanocrystals in an array, such as an ordered arrangement of nanocrystals in a crystalline lattice $[1,10,12-15]$ is useful for laser applications [10] or an optimal separation between the nanocrystals is useful for optoelectronic applications such as a photo-sensor [11]. Thus, it is desirable to have, not only a control on the size of the nanocrystals, but also an independent tunability of the inter-nanocrystal separation controlling the tunnelling of charge carriers between the nanocrystals. Here, we report a simple method to synthesize fixed-sized CdS nanocrystals but with varying inter-nanocrystal separations in a compacted assembly of powdered sample by varying the chain length of the passivating agent. The size and size dispersion of nanocrystals can be deduced by various methods such as UV-vis absorption, transmission electron microscopy, small angle x-ray scattering and x-ray diffraction. We make use of x-ray diffraction to simultaneously determine the size of the individual nanocrystals and the inter-nanocrystal separation by using wide- and low-angle regimes. 


\section{Experimental and methodology}

We synthesized CdS nanocrystals capped with three different capping agents, namely 1-thioglycerol, 1-hexanethiol and 1-decanethiol. A typical synthesis consists of dissolving $2.0 \mathrm{mmol}$ cadmium acetate and $2.39 \mathrm{mmol}$ capping agent (e.g. 1-thioglycerol) in $25 \mathrm{ml}$ methanol under inert atmosphere. $10 \mathrm{ml}$ of $0.2 \mathrm{M}$ sodium sulfide solution is then added to the reaction mixture dropwise and the reaction mixture is refluxed overnight. The nanocrystals are obtained by concentrating the solution and centrifuging to obtain solid powder samples.

X-ray diffraction (XRD) offers an easy and fast method to determine the size of the nanocrystals using the Debye-Scherrer equation

$$
L=\frac{0.9 \lambda}{B \cos \theta},
$$

where the coherence length, $L$, of the particle is related to the full-width at halfmaximum (FWHM), $B$, of the diffraction peak at $2 \theta$ recorded using x-rays of wavelength $\lambda$. For spherical nanocrystals, the coherence length is related to the diameter, $d$, by $d=\frac{4}{3} L$. This diameter is defined as the diameter of the core of the nanocrystal, which is crystalline in nature and does not include the thickness of the capping agent. Nanda et al [16] presented a method to determine the thickness of the capping agent using x-ray photoemission spectroscopy and theoretical modelling techniques. X-ray diffraction offers a relatively easy method to determine the inter-particle separation, as shown below.

Nanocrystals with very narrow size distribution, typically about $5 \%$, have a tendency to self-assemble into superstructures. These can be viewed as crystalline assemblies composed of individual nanocrystals at the lattice positions. Thus, they must give rise to diffraction at angles corresponding to the lattice spacing in these assemblies. If these nanocrystals, with diameter $d$ and thickness of the capping layer $t$, pack in a close packed arrangement then this spacing, $D$, corresponds to the (111) plane. Thus, for example, according to the Bragg's law,

$$
n \lambda=2 D \sin \theta
$$

the first-order diffraction peak at $2 \theta=2.2^{\circ}$ recorded with $\mathrm{Cu} \mathrm{K}$ radiation corresponds to a spacing of $40 \AA$ for the (111) plane. The diameter of the nanocrystal along with the capping thickness, $d+2 t$, is equal to $D$. Thus, the inter-particle separation, $2 t$, can be estimated using a combination of the wide-angle and low-angle $\mathrm{x}$-ray diffraction techniques.

\section{Results and discussion}

In figure 1, we show the wide-angle x-ray diffraction patterns for the three differently capped CdS nanocrystalline samples. Here we observe the striking similarity of the three diffraction patterns indicating that all the three nanocrystals have the same diameter. CdS bulk can exist either in the more stable wurtzite or the metastable zincblende structures; the corresponding XRD patterns are also shown in the figure. The extreme broadness of the diffraction peaks from the nanocrystals compared to that of the bulk system is indeed a reflection of the very small size of 
CdS nanocrystal assembly

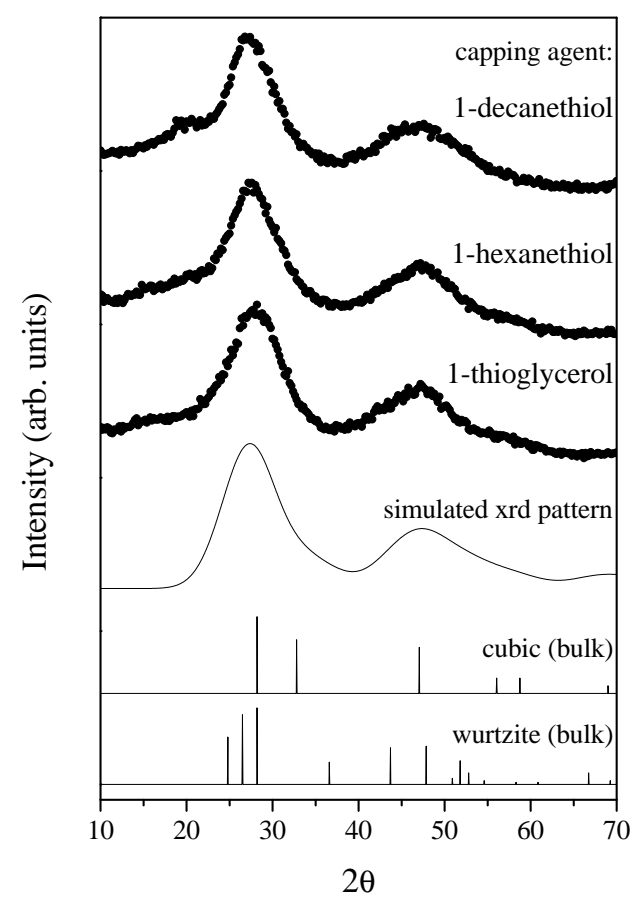

Figure 1. The wide-angle x-ray diffraction patterns for the CdS nanocrystals capped with 1-thioglycerol, 1-hexanethiol and 1-decanethiol. Shown below are the bulk wurtzite and cubic XRD patterns for CdS taken from the JCPDS-ICDD database. The cubic and wurtzite bulk patterns are broadened for $17.3 \AA$ diameter and added in the ratio $7: 3$ to obtain the best fit shown as solid line.

the nanocrystals. In order to extract the particle size from these results, we follow ref. [16] in reproducing the XRD pattern by convoluting the XRD pattern for the bulk CdS with the finite-size broadening function, given by the Debye-Scherrer formula, corresponding to a given size of the nanocrystal. This approach confirmed that the experimental XRD pattern from the nanocrystal samples are not compatible with a single crystal structure, either the wurtzite or the zincblende type. An addition of the XRD patterns for the cubic zinc blende and the hexagonal wurtzite structures in the ratio $7: 3$ convoluted with a broadening function corresponding to $17.3 \AA$ nanocrystal diameter, gives the best fit to the experimental data for all the three nanocrystal samples as shown in the figure by the simulated XRD pattern.

It is known that systems even without a long-range periodicity, such as amorphous solids or even liquids, give rise to peaks, though broad, in the diffraction pattern, reflecting a corresponding peak in the pair distribution function at the characteristic typical nearest-neighbour interatomic distance. Therefore, it is reasonable to expect a compacted powder sample of nanocrystals to exhibit a peak in the XRD pattern at the appropriate angle corresponding to the typical nearestneighbour nanocrystal-nanocrystal separation, with the entire nanocrystal playing the role of the atom in the example of the amorphous system we talked about. 


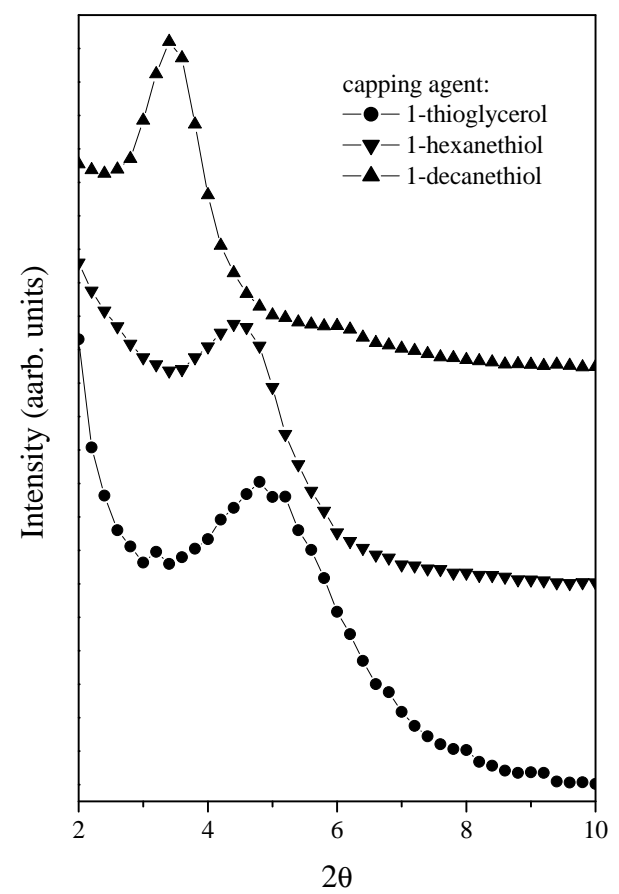

Figure 2. X-ray diffraction peaks at low angles for CdS nanocrystals capped with 1-thioglycerol, 1-hexanethiol and 1-decanethiol.

We indeed find these x-ray diffraction peaks and the results are shown in figure 2. These peaks appear at the low-angle regime of the XRD, as the characteristic length scales are considerably larger here than in the case of separation between the usual lattice planes. The low-angle peaks at $2 \theta=4.8^{\circ}, 4.4^{\circ}$ and $3.4^{\circ}$ correspond to an inter-nanocrystal separation of $18.4,20.0$ and $26.0 \AA$ for 1 thioglycerol, 1-hexanethiol and 1-decanethiol capping, respectively. It is interesting to note that the inter-nanocrystal separation systematically increases as the chain length of the capping agent increases though the size of the nanocrystals remain exactly the same, as evidenced by the wide-angle XRD result shown in figure 1 . It is reasonable to define the average effective thickness of the capping agent as half of the difference between the inter-nanocrystal separation determined from the low-angle diffraction peak and the individual nanocrystal diameter obtained from the wide-angle XRD patterns. This way we estimate the effective capping thickness on individual nanocrystals to be $0.55,1.35$ and $4.35 \AA$ for 1-thioglycerol, 1-hexanethiol and 1-decanethiol capping respectively. These numbers should be compared with the undistorted [17] chain lengths of 4.7, 9.5 and $13.7 \AA$ for 1thioglycerol, 1-hexanethiol and 1-decanethiol, respectively. There is a considerable difference between the effective capping thickness and the chain length; this suggests that either the long capping molecules fold up or point away from the surface normal, giving rise to a capping thickness considerably smallar than the chain length. 


\section{CdS nanocrystal assembly}

Table 1. The diameter determined from wide-angle XRD, $d$, diameter + thickness from the low-angle XRD, $d+2 t$, the average inter-particle separation, $2 t$, and the average chain length, $t$, for CdS nanocrystals capped with 1-thioglycerol, 1-hexanethiol and 1-decanethiol.

\begin{tabular}{lcccc}
\hline Capping agent & $d(\AA)$ & $d+2 t(\AA)$ & $2 t(\AA)$ & $t(\AA)$ \\
\hline 1-Thioglycerol & 17.3 & 18.4 & 1.1 & 0.55 \\
1-Hexanethiol & 17.3 & 20.0 & 2.7 & 1.35 \\
1-Decanethiol & 17.3 & 26.0 & 8.7 & 4.35 \\
\hline
\end{tabular}

In table 1 we list the sizes estimated from the wide-angle diffraction peak, $d$, low-angle diffraction peak, $d+2 t$, and the average separation between the nanocrystals, 2t. The thiol chain lengths, $t$, estimated using this method are smaller than the theoretical values 4.7, 9.5 and $13.7 \AA$ for 1-thioglycerol, 1-hexanethiol and 1decanethiol, respectively. We believe the chains in the nanocrystal assemblies tend to fold up to form a more dense structure and hence we observe shorter chain lengths.

\section{Conclusion}

In conclusion, we synthesized ordered CdS nanocrystals with various thiol capping agents. We studied the low-angle and wide-angle x-ray diffraction patterns for these thiol-capped CdS nanocrystals. This provides a simple means of estimating the inter-particle separation in ordered nanocrystal assemblies, which is necessary in order to use nanomaterials with capping agents in devices.

\section{Acknowledgements}

The authors would like to thank the Department of Science and Technology for financial support.

\section{References}

[1] C B Murray, C R Kagan and M G Bawendi, Ann. Rev. Mater. Sci. 30, 545 (2000)

[2] C B Murray, D J Norris and M G Bawendi, J. Am. Chem. Soc. 115, 8706 (1993)

[3] T Vossmeyer, L Katsikas, M Giersig, I G Popovic, K Diesner, A Chemseddine, A Eychmuller and H Weller, J. Phys. Chem. 98, 7665 (1994)

[4] J Nanda, S Sapra, D D Sarma, N Chandrasekharan and G Hodes, Chem. Mater. 12, 1018 (2000)

[5] M Bruchez Jr., M Moronne, P Gin, S Weiss and A P Alivisatos, Science 281, 2013 (1998)

[6] J Lee, V C Sundar, J R Heine, M G Bawendi and K F Jensen, Adv. Mater. 12, 1102 (2000)

[7] N Tessler, V Medvedev, M Kazes, S Kan and U Banin, Science 295, 1506 (2002)

[8] V L Colvin, M C Schlamp and A P Alivisatos, Nature (London) 370, 354 (1994) 


\section{Sameer Sapra and D D Sarma}

[9] K S Narayan, A G Manoj, J Nanda and D D Sarma, Appl. Phys. Lett. 74, 871 (1999)

[10] W U Hyunh, J J Dittmer and A P Alivisatos, Science 295, 2425 (2002)

[11] J Nanda, K S Narayan, B A Kuruvilla, G L Murthy and D D Sarma, Appl. Phys. Lett. 72, 1335 (1998)

[12] P J Thomas, P Saravanan, G U Kulkarni and C N R Rao, Pramana - J. Phys. 58, $371(2002)$

[13] C N R Rao, G U Kulkarni, P J Thomas and P P Edwards, Chem. Soc. Rev. 29, 27 (2000)

[14] P J Thomas, G U Kulkarni and C N R Rao, J. Phys. Chem. 104, 8138 (2000)

[15] A Courty, C Fermon and M-P Pileni, Adv. Mater. 13, 254 (2001)

[16] J Nanda, B A Kuruvilla and D D Sarma, Phys. Rev. B59, 7473 (1999)

[17] The theoretical values are calculated using the ACD/ChemSketch 5.0 Freeware 